\title{
ON SOLUTIONS TO SOME POLYNOMIAL CONGRUENCES IN SMALL BOXES
}

\author{
IGOR E. SHPARLINSKI
}

(Received 21 March 2013; accepted 15 June 2013; first published online 7 August 2013)

\begin{abstract}
We use bounds of mixed character sum to study the distribution of solutions to certain polynomial systems of congruences modulo a prime $p$. In particular, we obtain nontrivial results about the number of solutions in boxes with the side length below $p^{1 / 2}$, which seems to be the limit of more general methods based on the bounds of exponential sums along varieties.
\end{abstract}

2010 Mathematics subject classification: primary 11D79; secondary 11K38.

Keywords and phrases: multivariate congruences, distribution of points.

\section{Introduction}

There is an extensive literature investigating the distribution of solutions to the system of congruences

$$
F_{j}\left(x_{1}, \ldots, x_{n}\right) \equiv 0 \quad(\bmod p), \quad j=1, \ldots, m,
$$

with polynomials $F_{j}\left(X_{1}, \ldots, X_{n}\right) \in \mathbb{Z}\left[X_{1}, \ldots, X_{n}\right], j=1, \ldots, m$, in $m$ variables with integer coefficients, modulo a prime $p$; see $[4,5,8,11,12]$.

In particular, subject to some additional condition (related to the so-called $A$ number), Fouvry and Katz [5, Corollary 1.5] have given an asymptotic formula for the number of solutions to (1.1) in a box

$$
\left(x_{1}, \ldots, x_{n}\right) \in[0, h-1]^{n}
$$

for a rather small $h$. In fact, the limit of the method of [5] is $h=p^{1 / 2+o(1)}$.

Here we consider a very special class of systems of $s+1$ polynomial congruences

$$
x_{1} \cdots x_{n} \equiv a \quad(\bmod p)
$$

and

$$
c_{1, j} x_{1}^{k_{1, j}}+\cdots+c_{n, j} x_{n}^{k_{m, j}} \equiv b_{j} \quad(\bmod p), \quad j=1, \ldots, s,
$$

This work was supported in part by the ARC Grant DP130100237.

(c) 2013 Australian Mathematical Publishing Association Inc. 0004-9727/2013 \$16.00 
where $a, b_{j}, c_{i, j}, k_{i, j} \in \mathbb{Z}$, with $\operatorname{gcd}\left(a c_{i, j}, p\right)=1, i=1, \ldots, n, j=1, \ldots, s$, and $3 \leq$ $k_{i, 1}<\cdots<k_{i, s}$.

The interest in the systems of congruences (1.2) and (1.3) stems from the work of Fouvry and Katz [5], where a particular case of the congruence (1.2) and just one congruence of the type (1.3) (that is, for $s=1$ ) with the same odd exponents $k_{1,1}=\cdots=k_{n, 1}=k$ and $b_{1}=0$ is given as an example of a variety to which one of their main general results applies. In particular, in this case and for $k \geq 3, b_{1}=0$ (and fixed nonzero coefficients) we see that [5, Theorem 1.5] gives an asymptotic for the number of solutions with $1 \leq x_{i} \leq h, i=1, \ldots, n$, starting from the values of $h$ of size about $\max \left\{p^{1 / 2+1 / n}, p^{3 / 4}\right\} \log p$. Here we show that a different and more specialised treatment allows a significant lowering of this threshold, which now in some cases reaches $p^{1 / 4+\kappa}$ for any $\kappa>0$. Furthermore, this applies to the systems (1.2) and (1.3) in full generality and is uniform with respect to the coefficients.

More precisely, we use a combination of:

- $\quad$ the bound of mixed character sums to due to Chang [3];

- $\quad$ the result of Ayyad et al. [1] on the fourth moment of short character sums;

- $\quad$ the bound of Wooley [14] on exponential sums with polynomials.

We note that the classical Pólya-Vinogradov and Burgess bounds of multiplicative character sums (see [6, Theorems 12.5 and 12.6]), in combination with a result of Ayyad et al. [1], have been used in [9, 10] to study the distribution of the single congruence (1.2) in very small boxes, and thus go below the $p^{1 / 2}$-threshold.

Here we show that the recent result of Chang [3] enables us now to study a much more general case of the simultaneous congruences (1.2) and (1.3).

Throughout the paper, the implied constants in the symbols $O$ and « can depend on the positive parameter $\kappa$ and on the degrees $k_{i, j}$ in (1.2) and (1.3) as well as, occasionally, of some other polynomials involved. We recall that the expressions $A \ll B$ and $A=O(B)$ are each equivalent to the statement that $|A| \leq c B$ for some constant $c$.

\section{Character and exponential sums}

Let $\mathcal{X}_{p}$ be the set of multiplicative characters modulo $p$ and let $\mathcal{X}_{p}^{*}=\mathcal{X}_{p} \backslash\left\{\chi_{0}\right\}$ be the set of nonprincipal characters. We also write

$$
\mathbf{e}_{p}(z)=\exp (2 \pi i z / p)
$$

We appeal to [6] for a background on the basic properties of multiplicative characters and exponential functions, such as orthogonality.

The following bounds of exponential sums twisted with a multiplicative character have been given by Chang [3] for sums in arbitrary finite fields but only for intervals starting at the origin. However, a simple examination of the argument of [3] reveals that this is not important for the proof. 
Lemma 2.1. For any character $\chi \in \mathcal{X}_{p}^{*}$, a polynomial $F(X) \in \mathbb{Z}[X]$ of degree $k$ and integers $u$ and $h \geq p^{1 / 4+\kappa}$,

$$
\sum_{x=u+1}^{u+h} \chi(x) \mathbf{e}_{p}(F(x)) \ll h p^{-\eta}
$$

where

$$
\eta=\frac{\kappa^{2}}{4(1+2 \kappa)\left(k^{2}+2 k+3\right)} .
$$

We note that we do not impose any conditions on the polynomial $F$ in Lemma 2.1.

On the other hand, when $\chi=\chi_{0}$, we use the following very special case of the much more general bound of Wooley [14] that applies to polynomials with arbitrary real coefficients.

Lemma 2.2. For any polynomial $F(X) \in \mathbb{Z}[X]$ of degree $k>2$ with the leading coefficient $a_{k} \equiv 0(\bmod p)$, and any integers $u$ and $h$ with $h<p$,

$$
\sum_{x=u+1}^{u+h} \mathbf{e}_{p}(F(x)) \ll h^{1-1 / 2 k(k-2)}+h^{1-1 / 2(k-2)} p^{1 / 2 k(k-2)} .
$$

Clearly, Lemma 2.2 is nontrivial only for $h \geq p^{1 / k}$, which is actually the best possible range. Furthermore, in a slightly shorter range we have the following corollary.

Corollary 2.3. For any polynomial $F(X) \in \mathbb{Z}[X]$ of degree $k>2$ with the leading coefficient $a_{k} \not \equiv 0(\bmod p)$, and any integers $u$ and $h$ with $p^{1 /(k-1)} \leq h<p$,

$$
\sum_{x=u+1}^{u+h} \mathbf{e}_{p}(F(x)) \ll h^{1-1 / 2 k(k-2)} .
$$

We make use of the following estimate of Ayyad et al. [1, Theorem 1].

LEMMA 2.4. Uniformly over integers $u$ and $h \leq p$, the congruence

$$
x_{1} x_{2} \equiv x_{3} x_{4} \quad(\bmod p), \quad u+1 \leq x_{1}, x_{2}, x_{3}, x_{4} \leq u+h,
$$

has $h^{4} / p+O\left(h^{2} p^{o(1)}\right)$ solutions as $h \rightarrow \infty$.

We note that Lemma 2.4 is a essentially a statement about the fourth moment of short character sums; see [1, Equation (4)]. In fact, the next result makes it clearer.

COROLlary 2.5. Let $\rho(x)$ be an arbitrary complex valued function with

$$
|\rho(x)| \leq 1, \quad x \in \mathbb{R} .
$$


Uniformly over integers $1 \leq u \leq u+h<p$,

$$
\sum_{\chi \in \mathcal{X}_{p}}\left|\sum_{x=u+1}^{u+h} \rho(x) \chi(x)\right|^{4} \leq h^{4}+O\left(h^{2} p^{1+o(1)}\right),
$$

as $h \rightarrow \infty$.

Proof. Expanding the fourth power and changing the order of summation,

$$
\begin{aligned}
\sum_{\chi \in \mathcal{X}_{p}}\left|\sum_{x=u_{i}+1}^{u+h} \rho(x) \chi(x)\right|^{4} & =\sum_{\chi \in \mathcal{X}_{p}} \sum_{x_{1}, \ldots, x_{4}=u+1}^{u+h} \rho\left(x_{1}\right) \rho\left(x_{2}\right) \bar{\rho}\left(x_{3}\right) \bar{\rho}\left(x_{4}\right) \chi\left(x_{1} x_{2} x_{3}^{-1} x_{4}^{-1}\right) \\
& =\sum_{x_{1}, \ldots, x_{4}=u+1}^{u+h} \rho\left(x_{1}\right) \rho\left(x_{2}\right) \bar{\rho}\left(x_{3}\right) \bar{\rho}\left(x_{4}\right) \sum_{\chi \in \mathcal{X}_{p}} \chi\left(x_{1} x_{2} x_{3}^{-1} x_{4}^{-1}\right) .
\end{aligned}
$$

Using the orthogonality of characters, we write

$$
\begin{aligned}
\sum_{\chi \in \mathcal{X}_{p}}\left|\sum_{x=u+1}^{u+h} \rho(x) \chi(x)\right|^{4} & =(p-1) \sum_{\substack{x_{1}, \ldots, x_{4}=u+1 \\
x_{1} x_{2} \equiv x_{3} x_{4}(\bmod p)}}^{u+h} \rho\left(x_{1}\right) \rho\left(x_{2}\right) \bar{\rho}\left(x_{3}\right) \bar{\rho}\left(x_{4}\right) \\
& \leq(p-1) \sum_{\substack{x_{1}, \ldots, x_{4}=u+1 \\
x_{1} x_{2} \equiv x_{3} x_{4}(\bmod p)}}^{u+h} 1 .
\end{aligned}
$$

Applying Lemma 2.4, we derive the desired bound.

\section{Main result}

We are now able to present our main result. Let $\mathfrak{B}$ be a cube of the form

$$
\mathfrak{B}=\left[u_{1}+1, u_{1}+h\right] \times \cdots \times\left[u_{n}+1, u_{n}+h\right]
$$

with some integers $h, u_{i}$ with $1 \leq u_{i}+1<u_{i}+h<p, i=1, \ldots, n$. We denote by $N(\mathfrak{B})$ the number of integer vectors

$$
\left(x_{1}, \ldots, x_{n}\right) \in \mathfrak{B}
$$

satisfying (1.2) and (1.3) simultaneously.

As we have mentioned, the case of just one congruence (1.2) has been considered in $[9,10]$, so we always assume that $s \geq 1$ (and thus $n \geq 3$ ).

Let

$$
\begin{aligned}
& k=\min \left\{k_{i, j}: i=1, \ldots, n, j=1, \ldots, s\right\}, \\
& K=\max \left\{k_{i, j}: i=1, \ldots, n, j=1, \ldots, s\right\} .
\end{aligned}
$$

Recall that, due to our assumption, $K \geq k \geq 3$. 
THeOREM 3.1. For any fixed $\kappa>0$ and

$$
p>h \geq \min \left\{p^{1 / 4+\kappa}, p^{1 /(k-1)}\right\}
$$

we have

$$
N_{p}(\mathfrak{B})=\frac{h^{n}}{p^{s+1}}+O\left(h^{n} p^{-1-\eta(n-4)}+h^{n-2} p^{-\eta(n-4)}\right)
$$

where

$$
\eta=\frac{\kappa^{2}}{4(1+2 \kappa)\left(K^{2}+2 K+3\right)} .
$$

Proof. Using the orthogonality of characters, we write

$$
\begin{gathered}
N_{p}(\mathfrak{B})=\sum_{\left(x_{1}, \ldots, x_{n}\right) \in \mathfrak{B}} \frac{1}{p^{s}} \sum_{\lambda_{1}, \ldots, \lambda_{s}=0}^{p-1} \mathbf{e}_{p}\left(\sum_{j=1}^{s} \lambda_{j}\left(\sum_{i=1}^{n} c_{i, j} x_{i}^{k_{i, j}}-b_{j}\right)\right) \\
\times \frac{1}{p-1} \sum_{\chi \in \mathcal{X}_{p}} \chi\left(x_{1} \cdots x_{n} a^{-1}\right) .
\end{gathered}
$$

Hence, changing the order of summation,

$$
N_{p}(\mathfrak{B})=\frac{1}{(p-1) p^{s}} \sum_{\lambda_{1}, \ldots, \lambda_{s}=0}^{p-1} \mathbf{e}_{p}\left(-\sum_{j=1}^{s} \lambda_{j} b_{j}\right) \sum_{\chi \in \mathcal{X}_{p}} \chi\left(a^{-1}\right) \prod_{i=1}^{n} S_{i}\left(\chi ; \lambda_{1}, \ldots, \lambda_{s}\right),
$$

where

$$
S_{i}\left(\chi ; \lambda_{1}, \ldots, \lambda_{s}\right)=\sum_{x=u_{i}+1}^{u_{i}+h} \chi(x) \mathbf{e}_{p}\left(\sum_{j=1}^{s} \lambda_{j} c_{i, j} x^{k_{i, j}}\right), \quad i=1, \ldots, n .
$$

Separating the term $h^{n} /(p-1) p^{s}$, corresponding to $\chi=\chi_{0}$ and $\lambda_{1}=\cdots=\lambda_{s}=0$, we derive

$$
N_{p}(\mathfrak{B})-\frac{h^{n}}{(p-1) p^{s}} \ll \frac{1}{p^{s+1}}\left(R_{1}+R_{2}\right)
$$

where

$$
\begin{aligned}
& R_{1}=\sum_{\lambda_{1}, \ldots, \lambda_{s}=0}^{p-1} \sum_{\chi \in X_{p}^{*}} \prod_{i=1}^{n}\left|S_{i}\left(\chi ; \lambda_{1}, \ldots, \lambda_{s}\right)\right|, \\
& R_{2}=\sum_{\substack{\lambda_{1}, \ldots, \lambda_{s}=0 \\
\left(\lambda_{1}, \ldots, \lambda_{s}\right) \neq(0, \ldots, 0)}}^{p-1} \prod_{i=1}^{n}\left|S_{i}\left(\chi_{0} ; \lambda_{1}, \ldots, \lambda_{s}\right)\right| .
\end{aligned}
$$

To estimate $R_{1}$, we use Lemma 2.1 and write

$$
R_{1} \leq h^{n-4} p^{-\eta(n-4)} \sum_{\lambda_{1}, \ldots, \lambda_{s}=0}^{p-1} \sum_{\chi \in X_{p}^{*}} \prod_{i=1}^{4}\left|S_{i}\left(\chi ; \lambda_{1}, \ldots, \lambda_{s}\right)\right|
$$


Using the Hölder inequality and Corollary 2.5,

$$
\begin{aligned}
\sum_{\chi \in \mathcal{X}_{p}^{*}} \prod_{i=1}^{4}\left|S_{i}\left(\chi ; \lambda_{1}, \ldots, \lambda_{s}\right)\right| & \leq\left(\prod_{i=1}^{4} \sum_{\chi \in \mathcal{X}_{p}^{*}}\left|S_{i}\left(\chi ; \lambda_{1}, \ldots, \lambda_{s}\right)\right|^{4}\right)^{1 / 4} \\
& \ll h^{4}+h^{2} p^{1+o(1)}
\end{aligned}
$$

Therefore,

$$
R_{1} \ll h^{n} p^{s-\eta(n-4)}+h^{n-2} p^{s+1-\eta(n-4)} .
$$

Furthermore, for $R_{2}$ we use Corollary 2.3 to derive

$$
R_{2} \leq h^{(n-2)(1-1 / 2 K(K-2))} \sum_{\substack{\lambda_{1}, \ldots, \lambda_{s}=0 \\\left(\lambda_{1}, \ldots, \lambda_{s}\right) \neq(0, \ldots, 0)}}^{p-1} \prod_{i=1}^{2}\left|S_{i}\left(\chi_{0} ; \lambda_{1}, \ldots, \lambda_{s}\right)\right| .
$$

Using the Hölder inequality and the orthogonality of exponential functions (similarly to the proof of Corollary 2.5),

$$
\sum_{\substack{\lambda_{1}, \ldots, \lambda_{s}=0 \\\left(\lambda_{1}, \ldots, \lambda_{s}\right) \neq(0, \ldots, 0)}}^{p-1} \prod_{i=1}^{2}\left|S_{i}\left(\chi_{0} ; \lambda_{1}, \ldots, \lambda_{s}\right)\right| \leq\left(\prod_{i=1}^{2} \sum_{\lambda_{1}, \ldots, \lambda_{s}=0}^{p-1}\left|S_{i}\left(\chi_{0} ; \lambda_{1}, \ldots, \lambda_{s}\right)\right|^{2}\right)^{1 / 2} \ll p^{s} h .
$$

Thus

$$
R_{2} \ll h^{n-1-(n-2) / 2 K(K-2)} p^{s}
$$

Substituting the bounds (3.2) and (3.3) in (3.1),

$$
N_{p}(\mathfrak{B})-\frac{h^{n}}{p^{s+1}} \ll h^{n} p^{-1-\eta(n-4)}+h^{n-2} p^{-\eta(n-4)}+h^{n-1-(n-2) / 2 K(K-2)} p^{-1} .
$$

Clearly,

$$
4 \eta<\frac{1}{2 K(K-2)}
$$

Thus we see that

$$
p^{\eta(n-4)}<h^{4 \eta(n-4)}<h^{(n-2) / 2 K(K-2)} .
$$

Hence the second term always dominates the third term and the result follows.

\section{Comments}

Clearly, for any $\kappa>0, k \geq 5$ and $p>h \geq p^{1 / 4+\kappa}$, Theorem 3.1 implies that

$$
N_{p}(\mathfrak{B})=(1+o(1)) \frac{h^{n}}{p^{s+1}},
$$

as $p \rightarrow \infty$, provided that

$$
n \geq(s+1 / 2) \eta^{-1}+4
$$


For $k=3$ and 4 the range of Theorem 3.1 becomes $h \geq p^{1 / 2}$ and $h \geq p^{1 / 3}$, respectively. However, it is easy to see that using the full power of Lemma 2.2 instead of Corollary 2.3 one can derive nontrivial results in a wider range. Namely, for any $\kappa>0$ there exists some $\gamma>0$ (independent of $n$ and other parameters in (1.2) and (1.3)) such that, for $h \geq p^{1 / 3+\kappa}$ if $k=3$ and for $h \geq p^{1 / 4+\kappa}$ if $k=4$,

$$
N_{p}(\mathfrak{B})=\frac{h^{n}}{(p-1) p^{s}}+O\left(h^{(1-\gamma) n}\right) .
$$

We also recall that for polynomials of small degrees stronger versions of Lemma 2.2 are available; see [2] and references therein.

Note that the same method can be applied (with essentially the same results) to the systems of congruences where instead of (1.2) we have a more general congruence

$$
x_{1}^{m_{1}} \cdots x_{n}^{m_{n}} \equiv a \quad(\bmod p)
$$

for some integers $m_{i}$ with $\operatorname{gcd}\left(m_{i}, p-1\right)=1, i=1, \ldots, n$.

Moreover, we recall that the Weil bound [13, Appendix 5, Example 12] (see also [7, Ch. 6, Theorem 3]) and the standard reduction between complete and incomplete sums (see [6, Section 12.2]) imply that

$$
\sum_{x=u+1}^{u+h} \chi(G(x)) \mathbf{e}_{p}(F(x)) \ll p^{1 / 2} \log p,
$$

where $G(x)$ is a polynomial that is not a perfect power of any other polynomial in the algebraic closure $\overline{\mathbb{F}}_{p}$ of the finite field of $p$ elements. Thus for $h \geq p^{1 / 2+\kappa}$, using this bound instead of Lemma 2.1 allows us to replace (1.2) with the congruence

$$
G_{1}\left(x_{1}\right) \cdots G_{n}\left(x_{n}\right) \equiv a \quad(\bmod p)
$$

for arbitrary polynomials $G_{1}(X), \ldots, G_{n}(X) \in \mathbb{Z}[X]$ such that their reductions modulo $p$ are not perfect powers in $\overline{\mathbb{F}}_{p}$. In fact, even for $G_{1}(X)=\cdots=G_{n}(X)=X$ (that is, for the congruence (1.2)) this leads to a result which is sometimes stronger that those of [5] and Theorem 3.1.

\section{Acknowledgement}

The author is very grateful to Mei-Chu Chang for the confirmation that the main result of [3] applies to intervals in an arbitrary position.

\section{References}

[1] A. Ayyad, T. Cochrane and Z. Zheng, 'The congruence $x_{1} x_{2} \equiv x_{3} x_{4}(\bmod p)$, the equation $x_{1} x_{2}=$ $x_{3} x_{4}$ and the mean value of character sums', J. Number Theory 59 (1996), 398-413.

[2] K. D. Boklan and T. D. Wooley, 'On Weyl sums for smaller exponents', Funct. Approx. Comment. Math. 46 (2012), 91-107. 
[3] M.-C. Chang, 'An estimate of incomplete mixed character sums', in: An Irregular Mind, Bolyai Society Mathematical Studies, 21 (Springer, Berlin, 2010), 243-250.

[4] É. Fouvry, 'Consequences of a result of N. Katz and G. Laumon concerning trigonometric sums', Israel J. Math. 120 (2000), 81-96.

[5] É. Fouvry and N. Katz, 'A general stratification theorem for exponential sums, and applications', J. reine angew. Math. 540 (2001), 115-166.

[6] H. Iwaniec and E. Kowalski, Analytic Number Theory (American Mathematical Society, Providence, RI, 2004).

[7] W.-C. W. Li, Number Theory with Applications (World Scientific, Singapore, 1996).

[8] W. Luo, 'Rational points on complete intersections over $\mathbb{F}_{p}$ ', Int. Math. Res. Not. IMRN 1999 (1999), 901-907.

[9] I. E. Shparlinski, 'On the distribution of points on multidimensional modular hyperbolas', Proc. Japan Acad. Ser. A Math. Sci. 83 (2007), 5-9.

[10] I. E. Shparlinski, 'On a generalisation of a Lehmer problem', Math. Z. 263 (2009), 619-631.

[11] I. E. Shparlinski and A. N. Skorobogatov, 'Exponential sums and rational points on complete intersections', Mathematika 37 (1990), 201-208.

[12] A. N. Skorobogatov, 'Exponential sums, the geometry of hyperplane sections, and some Diophantine problems', Israel J. Math. 80 (1992), 359-379.

[13] A. Weil, Basic Number Theory (Springer, New York, 1974).

[14] T. D. Wooley, 'Vinogradov's mean value theorem via efficient congruencing, II', Duke Math. J. 162(4) (2013), 673-730.

IGOR E. SHPARLINSKI, Department of Computing, Macquarie University, Sydney, NSW 2109, Australia e-mail: igor.shparlinski@mq.edu.au 